\title{
Model Based Condition Monitoring for Reciprocating Aircraft Engines
}

\author{
Mair Andreas \\ Advanced Engineering \\ BRP-Powertrain \\ Gunskirchen, Austria \\ andreas.mair@brp.com
}

\author{
Thomas Thurner \\ Institute of Lightweight Design \\ Graz University of Technology \\ Graz, Austria \\ thomas.thurner@tugraz.at
}

\begin{abstract}
In this paper a novel method for detecting torque distortions on the power train system of reciprocating aircraft engines is proposed. Using only limited information of the system by acquisition of a single acceleration signal from the engine block our idea allows for robust and fast detection of severe faults like misfire in the engine's cylinders. Engine health condition is estimated from the acceleration data using modal analyses techniques based on the existing mechanical model. Results from detailed mechanical analyses of the power train system justify our single sensor approach. Our monitoring solution can be used as a cheap and easy misfire detection system as add-on safety application for existing and newly developed reciprocating aircraft engines.
\end{abstract}

Keywords: condition monitoring; aircraft engine; reciprocating engine; misfire detection; order analysis; modal analyses; on board diagnoses (OBD);.

\section{INTRODUCTION}

Small air planes often use reciprocating combustion engines for driving their propellers. One of the most severe failures in such aircrafts with reciprocating engines is related to combustion faults of single or multiple cylinders. This phenomenon is called misfire. It is clear that engine condition monitoring for robust and fast detection of such severe engine failures is very important for each aircraft to ensure safe and comfort flights. Although, most existing smaller aircrafts with reciprocating engines - especially older machines - do not have installed such monitoring and diagnosis systems.

The high level of the mechanical complexity of the reciprocating engines and their nonlinear dynamic behavior leads to only few commercially available monitoring solutions, with most of them being very complex analysis systems in terms of installation effort, computational needs and additional sensors most often having to be attached to or installed in the engine [5]. As a consequence, these systems are in most cases very expensive solutions with additional costs regarding system installation and its maintenance [6].

The idea behind this work is to develop a monitoring and diagnosis solution which is cheap and easily installable to existing piston aircraft engines, but still with robust and fast failure detection performance.

In contrast to existing methods our solution is based on information from a single acceleration sensor with very simple restrictions for attaching the sensor to the motor block. The sensor position is not critical; only the direction of the sensitive sensor axis needs to be aligned with respect to the primary engine case movement as discussed later.

The acquired acceleration information is then processed in a hierarchical signal processing scheme which makes it possible to detect failures in the mechanical power train system of such engines. To ensure robust detection of combustion failures order analysis of the acquired acceleration data is extended by a high level model-based estimation algorithm, which gives a quantitative measure of engine and cylinder health condition. Our solution enables a very fast and robust detection mechanism for identification of misfire and other severe combustion failures.

The following sections describe the mechanical model used for simulating the mechanical behavior of combustion aircraft engines and the principle of our condition monitoring system. Measurement results from experiments with real world aircraft engines are presented to verify the signal processing scheme and to evaluate the monitoring performance of our method. 


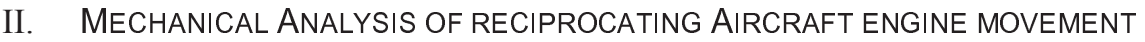

Mechanical models for the description of reciprocating aircraft engines differ significantly from conventional combustion engines as found in automotive vehicles. This is explained by the completely different operation mode of aircraft engines such as constant engine power close to the maximum available motor power compared with the best fuel consumption operating point over significantly larger duration times.

To describe the dynamic behavior of reciprocating aircraft engines and the observable effects of combustion failures on engine case movement a detailed mathematical description of the mechanical motor system has been developed. This model enables to simulate and analyze the interaction between engine case movement caused of the torque transmission and the in-cylinder pressure evolution for each active and defect cylinder.

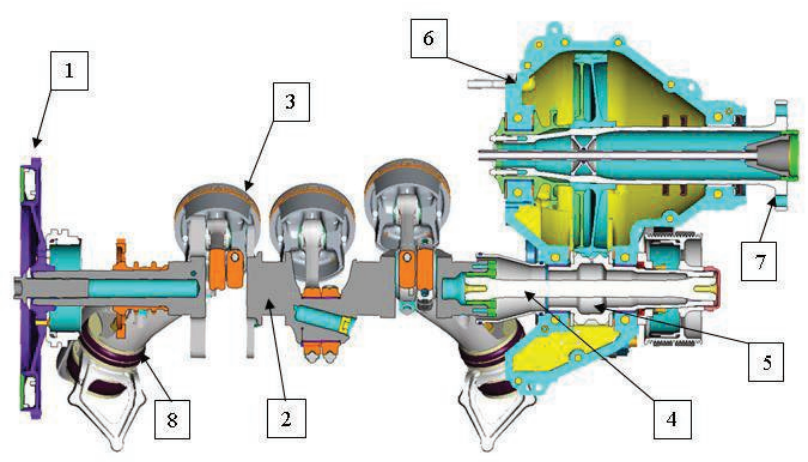

Figure 1: Left figure: Sectional representation of the power train system. Right figure: briefly engine model overview of the analytic model including the power train

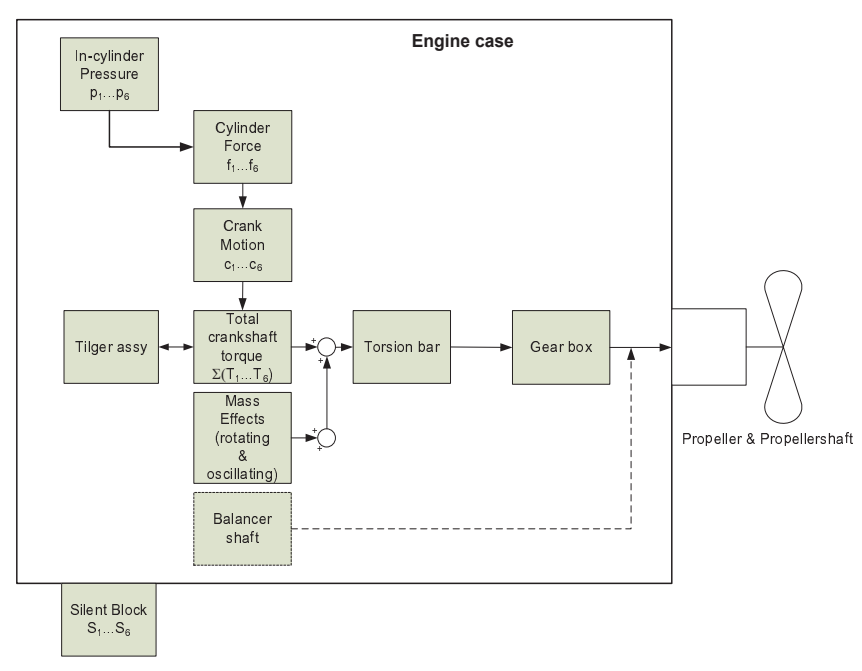
system, silent blocks and the propeller.

Figure 1 left hand side shows a sectional representation of the investigated engine with the hydro damper (1) on the left hand side, followed to the right by the crankshaft (2), the pistons (3), the torsion bar (4), the pinion shaft (5), the gearbox (6), and the propeller shaft (7). On the bottom the silent block (8) with the fixation is drawn as well. On the right hand side the developed analytic engine model in form of a diagram of the hierarchical engine block model based on the system shown in

Figure 1 left hand side was presents. The simulation of the engine case movement starts by an individual incylinder pressure calculation followed by estimating active gas forces. Based on knowledge of the evolution of gas forces in each cylinder, the total crankshaft torque can be computed by adding the acting forces of each cylinder. Due to the mechanical behavior of the crank mechanism the piston force has to be modeled by two forces (Figure 1 right hand):

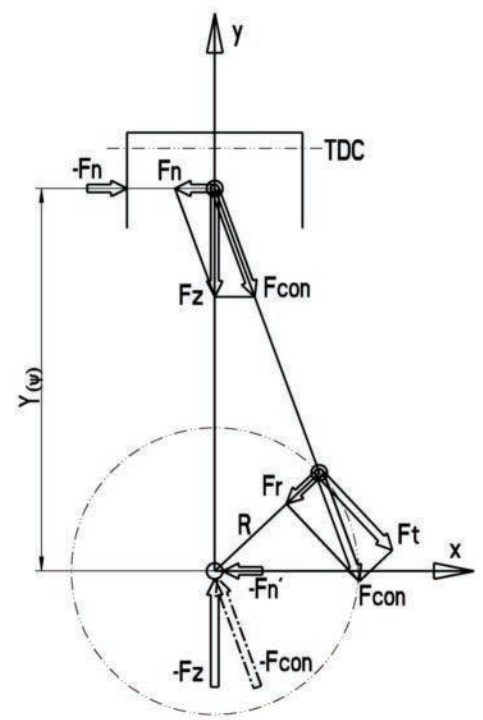

The piston force $F_{Z}$ is described in the cylinder's coordinate system, acting in up and down direction of the piston; the second force component is the transversal force $F_{n}$ of the piston force $F_{Z}$ and interacts with the barrel wall. The engine case movement is primarily caused by the force $F_{n}$ multiplied by the distance $y_{(\psi)}$ to the center of rotation of the crankshaft, which is called engine block torque $T_{e}$. For the simulation environment it is convenient to formulate the engine block torque as a function of the crank angle $\Psi$ and is denoted by $T_{e}=f(\Psi)$. It is easily shown that the engine case torque $T_{e}$ is equal to the negative crankshaft torque

$T_{c}=-T_{e}=-F_{n} \cdot y(\Psi)$.

Figure 2: Force components related to crank mechanism. 
The idea of our monitoring concept is to measure the engine case torque indirectly by acquisition of the engine block movement. Using the analytical mechanical power train model we recalculate the in-cylinder pressure for each individual cylinder. Since the case torque is invariant to the location on the engine the acceleration sensor can be mounted somewhere on the engine block. It is only required to have the sensitive axis aligned in proper direction to be able to detect the engine case movement.

Taking into account the individual ignition angle offsets for each cylinder, the total torque characteristics on the crankshaft can be calculated over a full engine combustion cycle. Following the block diagram of our developed analytical engine model presented in Figure 1, additional torque components from the crank mechanism also acting on the engine case due to rotating and oscillating masses have to be included as well. Next the torque transmission over the power train system must be calculated. The power train system is intended to transmit the torque to the propeller, and to reduce always existing engine vibrations. Thus the drive section needs to be considered as a dynamic system and the torque transmission needs to be calculated dynamically. The total engine case torque is then computed by a summation of the output torque of the drive system and the previously discussed influences from gas forces and mass phenomena. Finally, the effects of the silent blocks have to be calculated as well.

For all analyses using the developed simulation environment modal analysis techniques are applied, where signal power is analyzed in terms of engine rotations (orders). This kind of normalization of spectral signal analysis is used to obtain signal characteristics as a function of engine oscillation modes, allowing for easy comparison of signal characteristics for simulated and measured quantities.

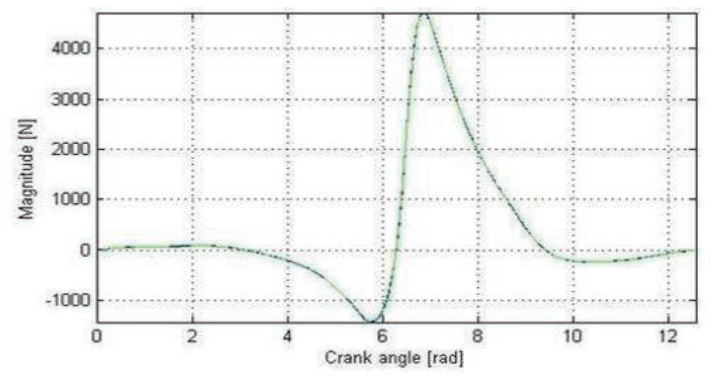

Figure 3: Simulation result for the piston transversal force component $F_{n}$ for a full engine cycle.

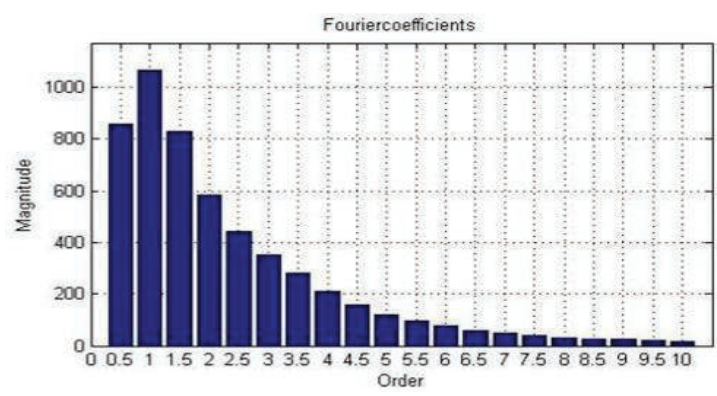

Figure 4: Modal analysis of the force $F_{n}$

Figure 3 presents a typical transversal gas force signal simulated for a single cylinder over a full engine cycle ( $4 \pi$ radians). These gas force signal evolution has been calculated from in-cylinder pressure curves obtained from realworld engine measurements. This plot shows the time evolution of the signal, whereas Figure 4 represents the corresponding modal signal power components. It can be seen that the main signal power is primarily concentrated within orders \#0.5 to \#2 with respect to a full engine rotation. Due to the fluctuation of the in-cylinder pressure amount in case of misfire the characteristics of $F_{n}$ is directly affected by this phenomenon. Following above discussions condition monitoring system for detecting misfire in single or multiple cylinders needs to incorporate signal information of at least these four orders.

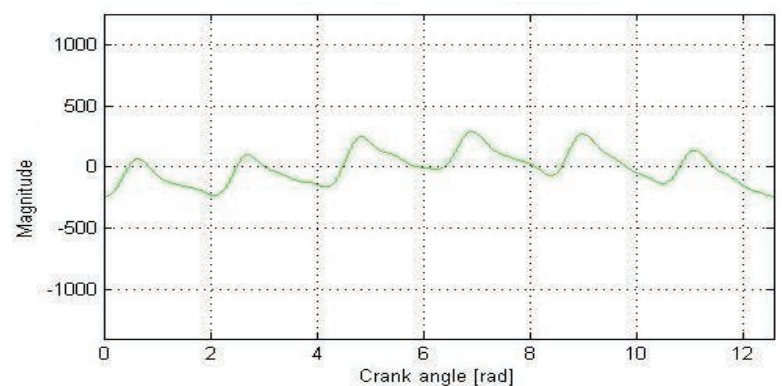

Figure 5: Simulated evolution of torque on the motor case plotted over crankshaft angle for a full combustion cycle of $4 \pi$ radiant for a 4 -stroke 6 cylinder reciprocating engine. Engine condition OK, each cylinder works properly.

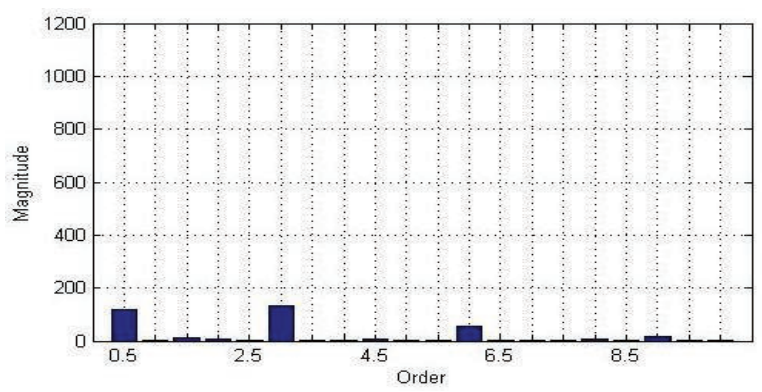

Figure 6: Order analysis for simulated engine case torque and normal combustion at all 6 cylinders. 
Figure 5 shows the simulated torque on the engine block for a fully functional engine. Each simulated ignition event at the cylinders leads to a single characteristic component in the torque signal, but with relative low signal amplitudes. The small differences in individual torque characteristics are originate from differences in the combustion process qualities of the different cylinders.

Figure 7 shows the simulated torque signal for a misfire event (combustion failure at cylinder 3 ). It can be seen that the fluctuations in torque on the engine case are dramatically increased when compared to a vital engine in Figure 5. As a consequence, engine case vibrations with significant vibrational amplitudes will be observable in case of combustion failures.

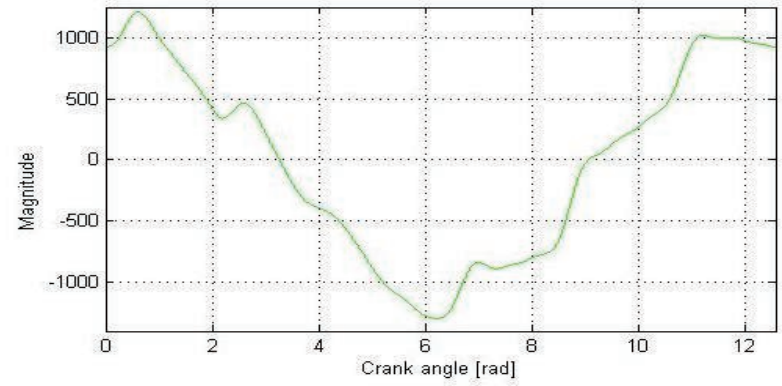

Figure 7: Simulated evolution of torque on the motor case plotted over crankshaft angle for a full combustion cycle of $4 \pi$ radiant for a 4-stroke 6 cylinder reciprocating engine. Engine conditions NOT OK: combustion failure at cylinder 3.

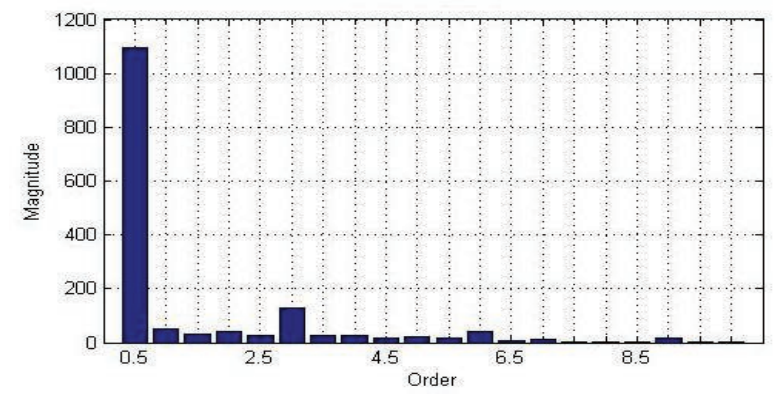

Figure 8: Order analysis of engine case torque for simulated misfire at cylinder 3.

Investigating the torque evolutions of Figure 5 and Figure 7 an order analysis with respect to engine rotation, leads to the analysis results as presented in Figure 6 and Figure 8. The most dominant motor order \#3 for a healthy engine (order 1 is related to a single engine rotation, which is half of a combustion cycle) is being outperformed by the engine oscillation mode \#0.5 in case of misfire at a single cylinder.

The most important results from developing a simulation environment and simulating the dynamic behavior of the overall power train system of the aircraft is a well-defined correlation between certain movements of the engine case and the combustion process of each individual cylinder: Our model predicts that a poor combustion process of one of the cylinders will result in abnormal engine torque on the crankshaft and the whole power train system and thus the introduction of additional or abnormal movements of the whole motor block.

The condition monitoring system described in the following section will utilize these findings to monitor each cylinder's actual combustion quality in a very robust manner.

\section{CONDITION MONITORING SYSTEM}

The condition monitoring system is based on information of engine case movement delivered by the single possibly low-cost acceleration sensor attached to the motor block. The exact location of the sensor with respect to the engine case is not essential for the monitoring process; only the sensor orientation with respect to the center of gravity of the engine is important.

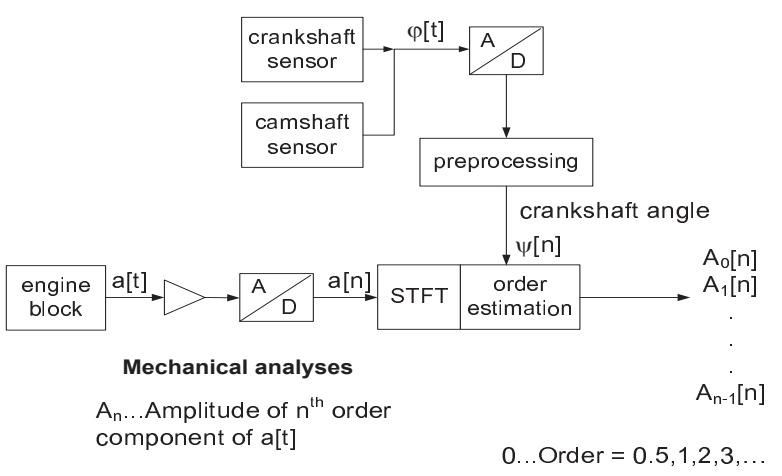

Figure 9: Signal processing scheme. Engine case movement is acquired by a single acceleration sensor. Order analysis based on known crankshaft rotation is used to evaluate the health condition for the engine and each cylinder. 
In addition to information of engine case torque delivered by the acceleration sensor, our signal processing chain also needs information about the actual crankshaft angle as provided by the built-in crankshaft sensor. Since crankshaft angle information is essential for engine operation controlled by the engine control unit (ECU) in modern reciprocating aircraft engines, such sensor data is always available for free. The first step in our data processing chain as sketched in Figure 9 shows the signal transition to the digital domain by anti-aliasing filtering and the following AVDconversion. The bandwidth of the engine acceleration sensor signal relevant for our condition monitoring is below $100 \mathrm{~Hz}$ leading to a sampling frequency of at least $200 \mathrm{~Hz}$ for practical aircraft drive scenarios. The digitized acceleration signal $a[n]$ is then fed into DFT-based order analysis, where the acceleration signal is analyzed in terms of the signal power distribution over relevant engine orders using the available crankshaft angle information.

To reduce leakage effects in these DFT-based analyses the used window length is chosen to be as close as possible to an even integer number of full rotations of the engine. Since the most important engine order to be evaluated for estimating cylinder health condition is order \#0.5 the smallest window length is given by the duration of two full engine turns (i.e. a full combustion cycle of the 4-stroke engine). The last step in the signal processing chain is the evaluation of the time evolution of signal power in certain engine orders. To be independent on engine speed a normalization based on the engine revolution is recommended (modal analysis).

The monitoring system uses overall signal power and the signal power of $0.5^{\text {th }}, 1^{\text {st }}$ and $1.5^{\text {th }}$ engine order from the order signal power estimation step as input variables. Based on the developed engine model the cylinder health condition is calculated from signal power distribution over orders 0.5 to 1.5 and the overall signal power.

\section{RESULTS}

In order to evaluate the accuracy and performance of the developed simulation environment and the found correlations between different physical quantities, real world measurements on reciprocating aircraft engines have been made in field tests with the possibility to switch off individual cylinders and monitoring of all relevant motor parameters. The switch-off process of individual cylinders has been accomplished by a shutdown of the corresponding injectors, leading to a very lean air-fuel mixture and then the combustion process stops immediately. During these experiments a single acceleration sensor has been attached to the test engine, a 6 cylinder 4-stroke reciprocating aircraft motor.

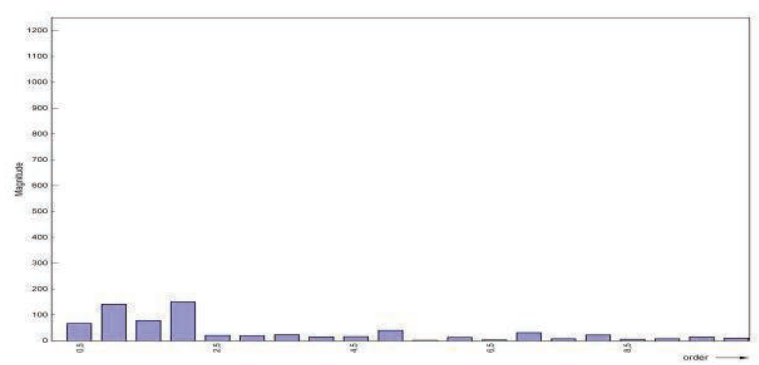

Figure 10: Order analysis for engine case acceleration signal from a healthy aircraft engine. Low motor orders show little vibrational amplitudes.

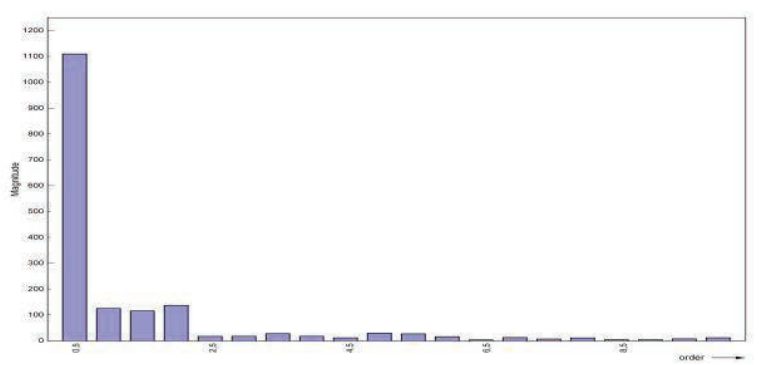

Figure 11: Evolution of signal power in individual engine orders from order analysis of real world engine case acceleration signals (aircraft engine with misfire). Six rotations after initiating defect in cylinder \#3.

Figure 10 shows the result of an order analysis for the given test engine over a full combustion cycle (healthy engine, two full motor rotations). The acquired overall acceleration signal has low amplitude, its power mainly contained within the four motor orders 0.5 to 2 . It can be seen that these results are in good agreement with order analyses from simulated data as provided in Figure 6.

The evolution of engine case signal power over engine orders for the entrance of misfire at a single cylinder is shown in Figure 11. The plot shows the order signal power spectrum 6 engine rotations after starting misfire, where almost all signal power is collected in order \#0.5

Finally Figure 12 (left hand side) represents the timely evolution of the overall signal power contained in engine orders \#0.5 to \#1.5 in comparison with the overall signal power for starting misfire at cylinder 3 and switching back to healthy engine condition after 50 rotations (the time is given in multiples of engine rotations). It can be seen that the overall signal power and especially the signal power of the $0.5^{\text {th }}$ engine order rises dramatically after beginning of misfire at a single cylinder. The consequences of such misfire immediately appear after only few rotations of the engine, a very fast response when compared to human reaction time: e.g. detection delay is far below than $100 \mathrm{~ms}$ for an engine speed of $6000 \mathrm{rpm}$.

As proofed in our analytic model the characteristics of the signal power versus time of the certain engine oscillation modes can be used to observe an engine health status. Due to the significant differences in the spectral signal power distribution when comparing good and a bad engine conditions a cylinder selective engine condition estimator can be realized. One possibility for fast and robust engine condition is the use of a fuzzy based system evaluating the spectral signal distribution in the low engine orders 0.5 to 1.5 as introduced in [8]. 
Figure 12, left diagram shows results from real world engine data (order analysis in Figure 11) analyzed by our fuzzy system. As long as the engine works without failures (no in-cylinder pressure loss) the engine health condition is ok (green area). Immediately after an engine failure (which is related to an in-cylinder pressure loss) occurs, the engine condition alters to the red area, engine not ok. As soon as the failure is gone, the engine health level goes back to the vital engine condition (green area).

During all field tests the simulated failures have been detected immediately and robustly [8]. If the manipulated injector has been switched on again the engine works in normal operation condition. This behavior of the engine system is in perfect agreement with simulation results from the developed mechanical description of such drive systems.
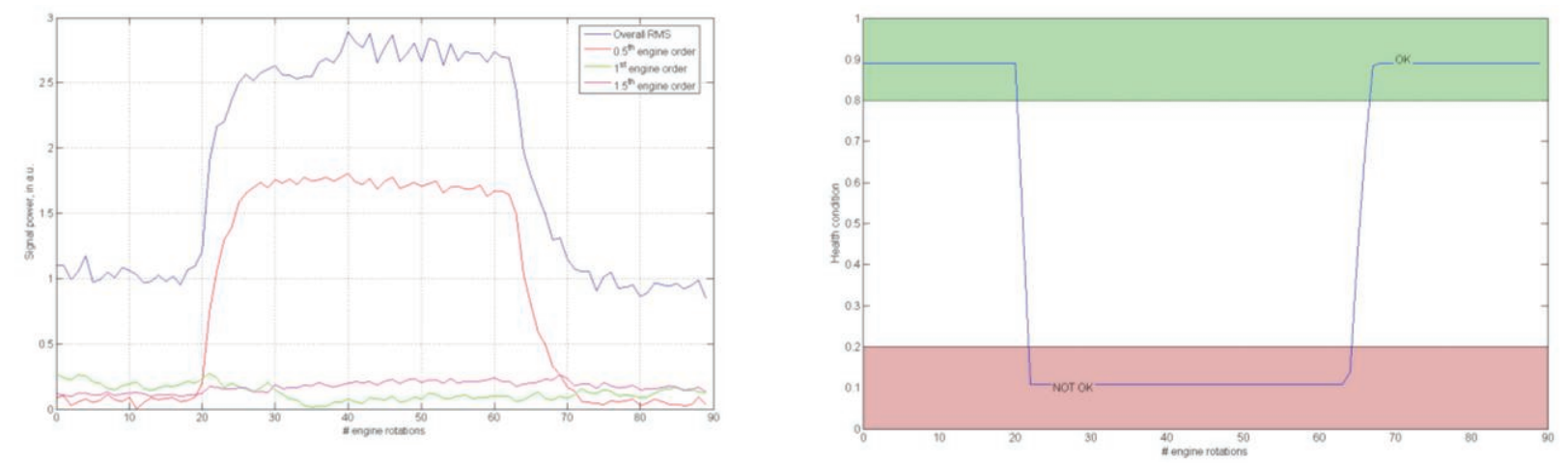

Figure 12: Left plot: Signal power of the engine orders 0.5 to 1.5 and the overall signal drawn versus engine cycles (two full engine rotations) after deactivating the injector for cylinder 3. Right plot: Fuzzy based calculated engine health status with engine health condition "OK" and "NOT OK".

\section{CONCLUSION}

Throughout this paper a powerful but easy possibility to monitor the condition of combustion processes in reciprocating aircraft engines has been proposed. Based on very detailed mechanical analyses an analytic model of the combustion engine including the aircraft's power train system has been developed. As an outcome of this work misfire events are found to lead to characteristic engine block movements, detectable by a single low cost acceleration sensor mounted on the engine. Real world measurements carried out on an experimental aircraft engine test rig are in good agreement with the results predicted by our simulations. Using these findings a fast and robust condition monitoring system for misfire detection has been developed and tested.

\section{References}

[1] Matteo Montani and Nicol'o Speciale. Multiple Misfire Identification by a Wavelet-Based Analysis of crankshaft Speed Fluctuation. International Symposium on Signal Processing and Information Technology, IEEE, 2006.

[2] C. Bohn, 0. Magnor and M. Schultalbers. State Observer based Analysis of Crankshaft Speed Measurements with Application to Misfire Detection. International Conference on Control and Automation (ICCA2005), 2005.

[3] Ruben Villarino and Johann F. Böhme. Misfire Detection in Automotive Engines Using Structure-Borne Sound.. SAE 2004 World Congress \& Exhibition, 2004.

[4] James M. Slicker. Knock and misfire detection system. Patent No.: US 6,546,328 B1, 2003.

[5] H. Heim and M. Mayer. Engine misfire detection and engine exhaust systems. Patent No.: WO 90/02871, 1990.

[6] RTCA, Inc. Environmental Conditions and Test Procedures for Airborne Equipment. USA, 2004.

[7] Michael Wagner, Jürgen Förster and Johann F. Böhme. In-Cylinder Pressure Estimation From Structure-Borne Sound. SAE 2000 World Congress, 2000.

[8] Andreas Mair and Thomas Turner. Condition Monitoring for Reciprocating Aircraft Engines using Fuzzy Logic. Computational Intelligence for Measurement Systems and Application (CIMSA), IEEE, 2010. 